\title{
ARTICLE
}

Received 27 Jun 2014 | Accepted 5 Nov 2014 | Published 9 Jan 2015 DOl: 10.1038/ncomms6763

\section{Tandem intercalation strategy for single-layer nanosheets as an effective alternative to conventional exfoliation processes}

Sohee Jeong ${ }^{1, \star}$, Dongwon Yoo ${ }^{1, \star}$, Minji Ahn ${ }^{1}$, Pere Miró ${ }^{2}$, Thomas Heine ${ }^{2} \&$ Jinwoo Cheon ${ }^{1}$

Simple and effective generation of transition metal chalcogenides (TMCs) in a single-layer form has been a challenging task. Here we present a tandem molecular intercalation (TMI) as a new exfoliation concept for producing single-layer TMCs from multi-layer colloidal TMC nanostructures in solution phase. TMI requires tandem Lewis base intercalates, where short 'initiator' molecules first intercalate into TMCs to open up the interlayer gap, and the long 'primary' molecules then bring the gap to full width so that a random mixture of intercalates overcomes the interlayer force. Spontaneous exfoliation then yields single-layer TMCs. The TMI process is uniquely advantageous because it works in a simple one-step process under safe and mild conditions (that is, room temperature without sonication or $\mathrm{H}_{2}$ generation). With the appropriate intercalates, we have successfully generated single-layer nanostructures of group IV $\left(\mathrm{TiS}_{2}, \mathrm{ZrS}_{2}\right)$, group $\mathrm{V}\left(\mathrm{NbS}_{2}\right)$ and $\mathrm{VI}\left(\mathrm{WSe}_{2}, \mathrm{MoS}_{2}\right) \mathrm{TMCs}$.

\footnotetext{
${ }^{1}$ Department of Chemistry, Yonsei University, Seoul 120-749, Korea. ${ }^{2}$ Engineering and Science, Jacobs University Bremen, 28759 Bremen, Germany. ${ }^{\star}$ These authors contributed equally to this work. Correspondence and requests for materials should be addressed to J.C. (email: jcheon@yonsei.ac.kr).
} 
$\mathrm{T}$ wo-dimensional (2D) layered materials including transition metal chalcogenides (TMCs) with strong in-plane covalent bonds and weak interlayer van der Waals interactions exhibit a wide range of interesting anisotropic phenomena from charge transport to catalytic and optical properties $^{1-9}$. Similar to graphene, such interesting effects are centred on single-layer TMCs and, as an example, when $\mathrm{MoS}_{2}$, an indirect band gap semiconductor in bulk, is thinned to a single layer, a strong photoluminescence is observed due to the indirectto-direct band gap change ${ }^{6,7}$.

Single-layer or few-layer nanosheets can be obtained from bulk TMCs by several exfoliation techniques such as dry mechanical cleavage with Scotch tape $e^{10,11}$ or solution-based exfoliation processes ${ }^{12-26}$. Intercalation of alkali metals by using elemental metals ${ }^{12,13}$ (for example, potassium) or organo-alkali compounds (for example, butyllithium ${ }^{14-16}$ and more recently sodium naphthalenide ${ }^{17}$ ) has been a widely used method for the exfoliation of layered materials. Such exfoliation and disintegration methods with $\mathrm{K}$, air and $\mathrm{EtOH}-\mathrm{H}_{2} \mathrm{O}$ along with sonication of layered flakes have successfully generated monolayer nanoparticles of $\mathrm{WS}_{2}, \mathrm{BN}$ and graphene $e^{9,12,13}$. Although quite effective, the use of reactive chemicals during exfoliation conditions and $\mathrm{H}_{2}$ generation through multiple exfoliation processes has drawbacks ${ }^{17}$. While the electrochemical exfoliation method has been developed as a faster and controllable exfoliation protocol, complicated electrochemical set-ups present challenges for the mass production of single-layer nanosheets ${ }^{18-21}$. Alternatively, a sonication method in the presence of solvents ${ }^{22,23}$ such as $\mathrm{N}$-methyl-pyrrolidone has been demonstrated for the successful exfoliation of wide range of TMCs, but the products are often mixed with single- and multi-layer TMCs with potential degradation of samples due to the harsh sonication conditions required to overcome the interlayer interactions ${ }^{24-26}$. As stated here, current methodologies have their own advantages and disadvantages, but none of them are yet perfect ${ }^{17,26}$, although it is true that these are effective for large-size layered nanostructures. The development of mild exfoliation strategies that can circumvent the key harsh process conditions (for example, sonication and $\mathrm{H}_{2}$ gas formation) has been challenging and, therefore, new and better routes to obtain single-layer TMCs in a simple, efficient and reproducible manner are currently being pursued. In this study, we introduce the 'tandem molecular intercalation (TMI)' process as a mild and effective exfoliation strategy for single-layer 2D TMC nanosheets from multi-layer colloidal TMC nanostuctures. Our TMI process utilizes two different Lewis base intercalates for the initiative and primary roles. Relatively short length 'initiator' Lewis base molecules are introduced into the interlayer space of $2 \mathrm{D}$ TMCs to start the gap-widening process, and a simultaneous influx of 'primary' intercalate molecules with longer chains creates randomly mixed bilayers of intercalates to overcome the interlayer interactions and eventually generate single-layer nanosheets (Fig. 1). Although the host-guest chemistry for the intercalation of Lewis bases into bulk TMCs has been well documented ${ }^{27,28}$, its successful extension to an exfoliation strategy has not been demonstrated yet.

\section{Results}

Intercalation of alkylamine in $\mathrm{TiS}_{\mathbf{2}}$ colloidal nanostructure. We first examined the group IV $\mathrm{TiS}_{2}$ colloidal nanostructure $(60 \mathrm{~nm}$ in diameter with a dozen layers) with an interlayer distance of $5.7 \AA$ and with each single-layer $\mathrm{TiS}_{2}$ comprising a S-Ti-S tri-atomic plate of 1T-type $\mathrm{CdI}_{2}$ structure ${ }^{29}$ (Fig. 2a). The intercalation and gap-widening capability of Lewis base molecular intercalates were investigated depending on the alkyl chain length of primary amines such as propyl-, butyl- and hexyl-amine (Fig. 2b $)^{29,30}$. Here, an excess amount of intercalate is added to $\mathrm{TiS}_{2}$ nanoparticle in dimethyl sulphoxide (DMSO) at room temperature, and the reaction mixture is stirred for $30 \mathrm{~min}$ to $5 \mathrm{~h}$ depending on the chain length of the intercalate molecules. Subsequently, the colloidal suspension is precipitated by centrifugation and washed with chloroform. The initial interlayer distance of pristine $\mathrm{TiS}_{2}$ is $5.7 \AA$, but after intercalation with propyl- butyl- and hexyl-amine, it increases to $9.5,10.5$ and $14.3 \AA$, respectively, according to TEM analysis (Fig. 2c-f).

XRD analysis also confirms the widening interlayer distance (Fig. 2h). Interlayer gap dependent $c$ axis peaks, such as (001), (002) and (003), are shifted to lower angles as the intercalate size increases. Pronounced effects are observed for the (001) peak position, where the $2 \theta$ angle changes from $15.5^{\circ}$ to $9.0^{\circ}, 8.7^{\circ}$ and $6.3^{\circ}$ for propylamine, butylamine and hexylamine, respectively. From the $2 \theta$ values, the interlayer distances are calculated to be 5.7, 9.8, 10.2 and $14.0 \AA$, and the expansion of the $c$ axis is $4.1 \AA$ for propylamine, $4.5 \AA$ for butylamine and $8.3 \AA$ for hexylamine (Fig. 2j), respectively. This (001) peak shift trend and TEM analysis confirm that the interlayer gap widening of $\mathrm{TiS}_{2}$ is proportional to the alkylamine length. As the lengths of propyl-, butyl- and hexyl-amine are $\sim 4.1,5.1$ and $7.7 \AA$ (Fig. 2i), respectively, the observed expansion of the interlayer gap suggests a head-to-tail monolayer arrangement of alkylamines between the layers $^{31}$.

This alkylamine intercalation process proceeds through a monolayer arrangement at the initial stage, but after lengthened intercalation time, the intercalates start to adopt a bilayer arrangement between the layers. With the use of propylamine, the $2 \theta$ angle of the (001) peak position of $\mathrm{TiS}_{2}$ shifts from $15.5^{\circ}$ to $8.9^{\circ}$ at $45 \mathrm{~min}$ and $6.4^{\circ}$ at $7 \mathrm{~h}$ (Fig. 3a). At the intermediate time of $3 \mathrm{~h}$, the two peaks at $8.9^{\circ}$ and $6.4^{\circ}$ are simultaneously observed. These XRD results suggest that the $\mathrm{TiS}_{2}$ interlayer distance of $9.9 \AA$ corresponds to a monolayer of propylamine, which subsequently changes to a bilayer formation (13.8 ̊̊) through an intermediate state $(3 \mathrm{~h})$ (Fig. 3b). The intercalation modes of alkylamines follow monolayer to bilayer arrangements, and the interlayer gap widening can be controlled using different alkylamine intercalates. However, none of the layered $\mathrm{TiS}_{2}$ show spontaneous exfoliation even though the London-dispersioncorrected DFT calculation estimates a critical interlayer distance of $\sim 11 \AA$ to overcome the interlayer van der Waals interaction between two layers of pristine $\mathrm{TiS}_{2}$ (Fig. 3c). Our observation of non-exfoliation phenomenon is presumably due to the additional energy needed to overcome partially or fully interdigitated interactions between the alkyl chains of the intercalates (Fig. 3b) $)^{30,32,33}$.

Exfoliation of TMCs via TMI method. Here, we devised the TMI concept, where two different lengths of intercalates are utilized for the exfoliation of layered nanostructures. This TMI process has distinctive features, namely a short 'initiator' alkylamine first expands the interlayer gap for efficient intercalation of the 'primary' long alkylamine; the difference in the chain length, then, generates a bilayer arrangement with empty space between intercalates to reduce van der Waals force between them; and finally spontaneous exfoliation occurs (Fig. 4a). We examined the TMI concept for the exfoliation of multi-layer $\mathrm{TiS}_{2}$. By adding propylamine and hexylamine to colloidal $\mathrm{TiS}_{2}$ nanostructure in DMSO at room temperature, the intercalation of propylamine is observed with an expanded interlayer distance of $9.5 \mathrm{~A}$ in $30 \mathrm{~min}$ (Fig. 4b), and the presence of intercalated propylamine is 


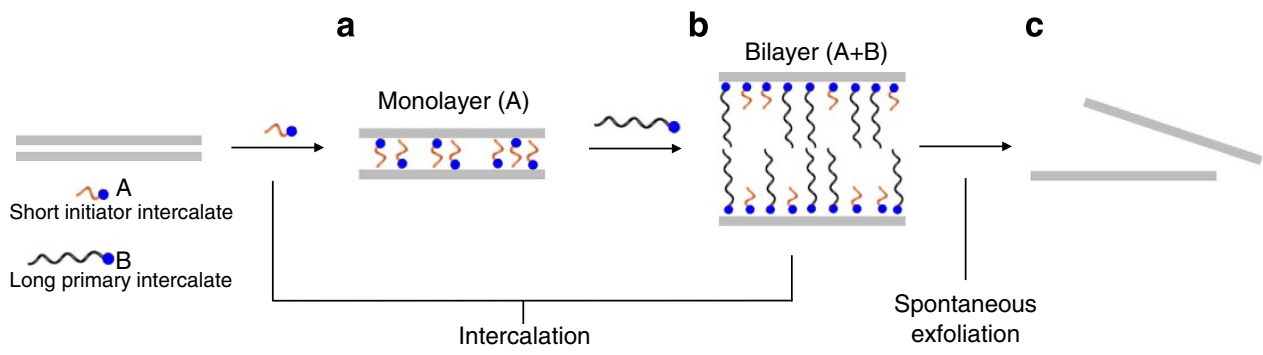

Figure 1 | Tandem molecular intercalation process for exfoliation of TMCs. The TMI process incorporates two different Lewis base intercalates. (a) Short 'initiator' intercalates (orange tail) are intercalated first into the interlayer gap, and (b) long 'primary' intercalates (black tail) then enter to form randomly mixed bilayers of intercalates and widen the interlayer gap. (c) Finally, TMCs with bilayer intercalates are spontaneously delaminated to single-layer nanosheets.

a

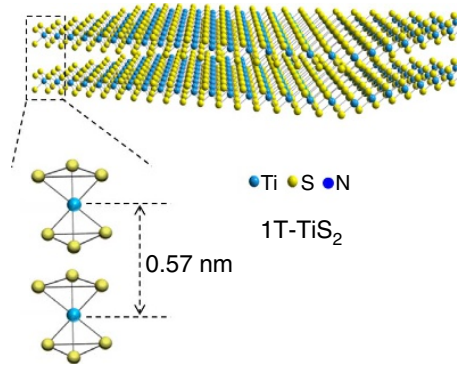

h

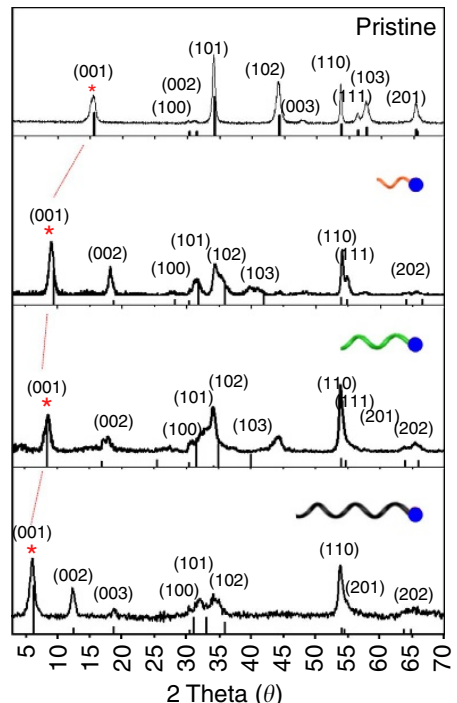

b

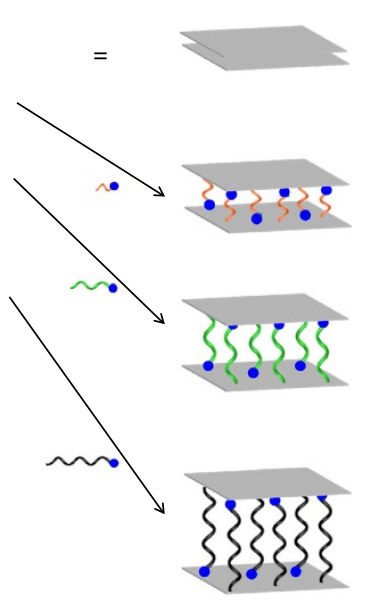

C

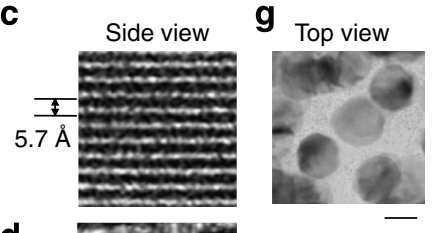

i

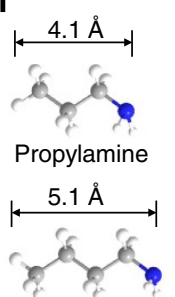

Butylamine

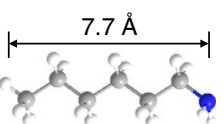

Hexylamine d

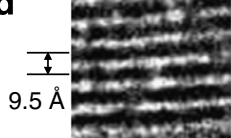

e

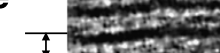

$10.5 \AA$

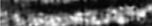

Shaselante:

$\mathbf{f}$
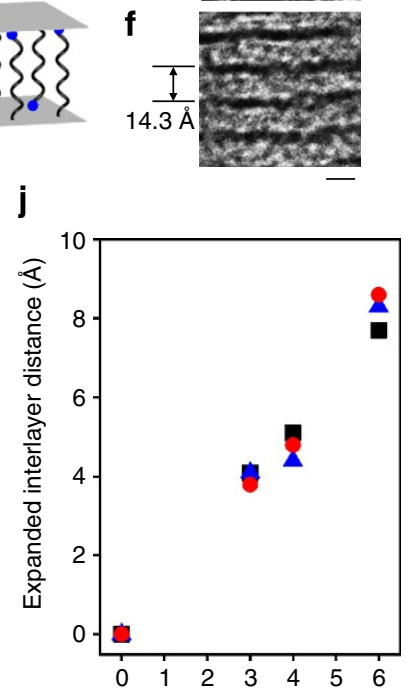

Number of $C$ atoms in alkyl chain

Figure 2 | Intercalate size-dependent interlayer expansion of multi-layer $\mathbf{T i S}_{\mathbf{2}}$. (a) Visualization of $\mathrm{TiS}_{2}$ using ball and stick model to illustrate the 2D layered structure. (b) Schematic illustration of interlayer distance change of $\mathrm{TiS}_{2}$ by intercalating alkylamine. Side view of layers in $\mathrm{TEM}$ images (c) before intercalation and after intercalation of (d) propylamine, (e) butylamine and (f) hexylamine. Scale bar, $1 \mathrm{~nm}$. (g) Top-view TEM image of multi-layer $\mathrm{TiS}_{2}$ before exfoliation. Scale bar, $50 \mathrm{~nm}$. (h) XRD patterns of pristine $\mathrm{TiS}_{2}$ and intercalated $\mathrm{TiS}_{2}$ with propylamine, butylamine and hexylamine. Vertical lines indicate reference peaks of bulk and expanded $\mathrm{TiS}_{2}$ (JCPDS \#15-0853). (i) Calculated length between nitrogen and terminal hydrogen of propylamine $(4.1 \AA)$, butylamine $(5.1 \AA)$ and hexylamine $\left(7.7 \AA\right.$ ) by ChemBio $3 D^{\circledR}$ Ultra program. (j) Expanded interlayer distance of TiS 2 plotted against the number of carbon $(C)$ atoms in alkyl chain of intercalate (black square, calculated value; blue triangle, value observed by XRD; red circle, value observed by TEM).

confirmed by ${ }^{1} \mathrm{H}-\mathrm{NMR}$. Then, further expansion of the layer distance to $21 \AA$ is observed in $6 \mathrm{~h}$ (Fig. $4 \mathrm{c}$ ), and the coexistence of propylamine and hexylamine is confirmed by ${ }^{1} \mathrm{H}$-NMR. At this stage, the layers spontaneously begin to separate, and single-layer
$\mathrm{TiS}_{2}$ nanosheets are obtained. Figure 4d,e show TEM images of single-layer $\mathrm{TiS}_{2}$ nanosheets after exfoliation. A top-view highresolution TEM (HRTEM) image of a single-layer $\mathrm{TiS}_{2}$ nanosheet shows lattice fringes with interplanar spacings of 2.9 and $1.7 \AA$, 
a

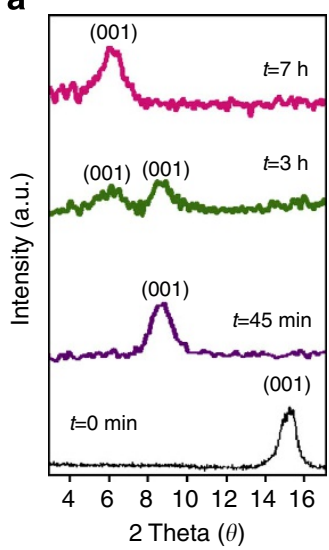

b

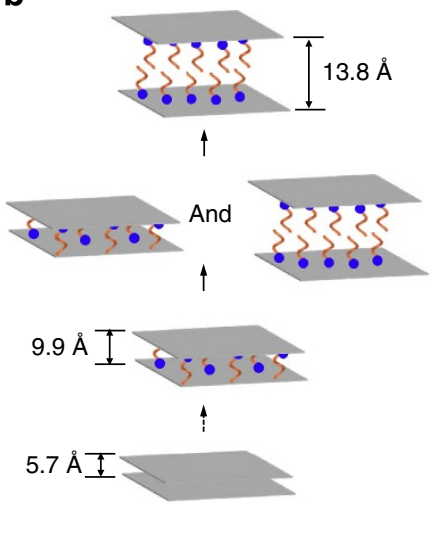

C

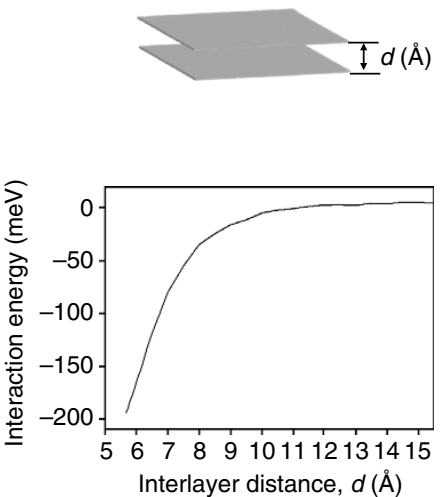

Figure 3 | Time-dependent interlayer distance change of multi-layer $\mathbf{T i S}_{\mathbf{2}}$ with propylamine. (a) XRD patterns of intercalated TiS 2 at 0 min, 45 min, $3 \mathrm{~h}$ and $7 \mathrm{~h}$ after treatment of propylamine. (b) Illustration of interlayer distance change of $\mathrm{TiS}_{2}$ by intercalating propylamine. (c) Interlayer interaction energy calculated by density functional theory at the PBE-D3-BJ level. The critical interlayer distance for pristine $\mathrm{TiS}_{2}$ exfoliation is estimated to be $\sim 11.0 \AA$, where the interaction energy is close to $\sim k_{\mathrm{B}} \mathrm{T}$.

a
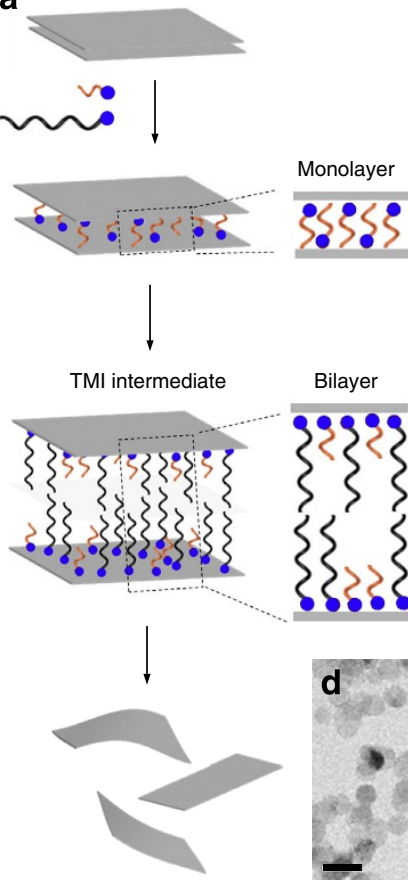

b
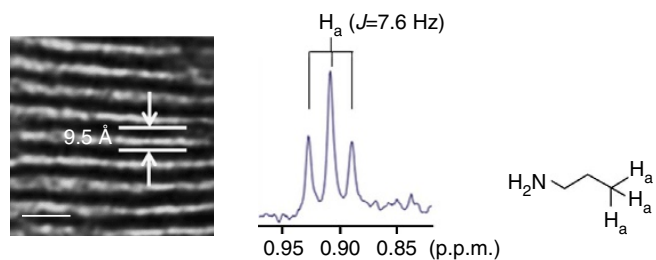

C

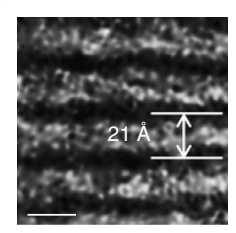

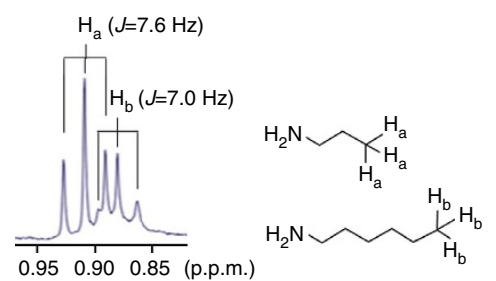

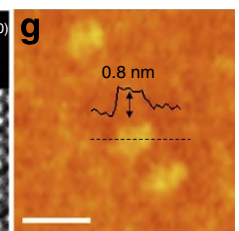

Figure 4 | Exfoliation of multi-layer TiS $_{\mathbf{2}}$ nanostructure by TMI process. (a) Illustration of tandem molecular intercalates (TMI) process. Side-view TEM image and ${ }^{1} \mathrm{H}$-NMR spectrum of $\mathrm{TiS}_{2}$ intercalated with (b) propylamine and (c) both propylamine and hexylamine. (d) Top-view and (e) magnified TEM images of $\mathrm{TiS}_{2}$ after exfoliation. (f) HRTEM image and FFT pattern (inset) of a single-layer $\mathrm{TiS}_{2}$ nanosheet. (g) AFM image of single-layer TiS 2 nanosheets. Scale bars, $2 \mathrm{~nm}$ in $\mathbf{b}$ and $\mathbf{c} ; 100 \mathrm{~nm}$ in $\mathbf{d}$ and $\mathbf{g} ; 20 \mathrm{~nm}$ in $\mathbf{e} ; 1 \mathrm{~nm}$ in f.

corresponding to the (100) and (110) planes of the $\mathrm{TiS}_{2}$, respectively (Fig. 4f). The single-layer thickness of $8.0 \AA$ is confirmed by AFM analysis (Fig. 4g). Additional XRD analysis shows single peak of (110) plane at $53.7^{\circ}$ without any peaks in $c$ axis direction, which confirms that final products are fully exfoliated single-layer $\mathrm{TiS}_{2}$ (Supplementary Figs $1 \mathrm{a}$ and $2 \mathrm{~b}$ ). Such experimental observations are in good agreement with the calculations based on density-functional tight-binding theory, which confirms that mixed intercalates of different chain lengths decrease the total interaction energy below $2 k_{\mathrm{B}} \mathrm{T}$ to have an effective exfoliation (Supplementary Fig. 3, Supplementary Tables 1-3).
Our tandem molecular exfoliation method can be applied to other colloidal nanoparticles of group IV, V and VI TMCs, such as $\mathrm{ZrS}_{2}, \mathrm{NbS}_{2}$ and $\mathrm{WSe}_{2}$. First, the exfoliation of multi-layer $\mathrm{ZrS}_{2}$ nanostructure (17 $\mathrm{nm}$ in lateral size and three layers) is examined (Fig. 5a,b). Although less explored than group VI counterparts, group IV TMCs also exhibit interesting single-layer properties such as enhanced thermoelectric property and electrical conductivity for $\mathrm{TiS}_{2}$ and strain-driven indirect-to-direct band gap transition of $\mathrm{ZrS}_{2}$ (refs 34-36). Similar to the case of $\mathrm{TiS}_{2}$, propylamine and hexylamine are added to $\mathrm{ZrS}_{2}$ nanostructure in DMSO with stirring at room temperature. After $4 \mathrm{~h}$, the interlayer distance is expanded from $5.8 \AA$ (Fig. 5b) to $20.5 \AA$ (Fig. 5c). 

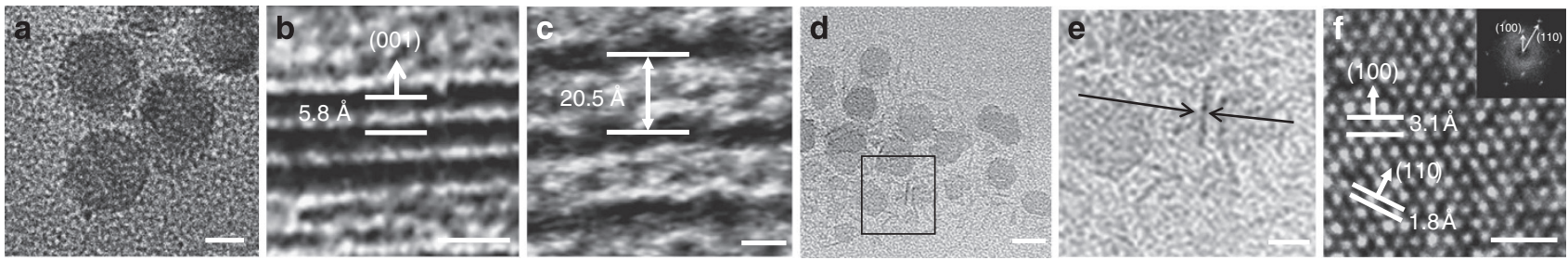

Figure 5 | Exfoliation of multi-layer $\mathbf{Z r S}_{\mathbf{2}}$ nanostructure. (a) Top-view and (b) side-view TEM image of three-layer $\mathrm{ZrS}_{2}$ nanostructure before exfoliation. (c) Side-view TEM image of propylamine and hexylamine intercalated $\mathrm{ZrS}_{2}$. (d) TEM image and (e) magnified TEM image of the single-layer $\mathrm{ZrS}_{2}$ nanosheets in the box of $\mathbf{d}$. A side-view of the single-layer $\mathrm{ZrS}_{2}$ nanosheet between the arrows. (f) HRTEM image and FFT pattern (inset) of a single-layer $\mathrm{ZrS}_{2}$ nanosheet. Scale bars, $10 \mathrm{~nm}$ in $\mathbf{a}$ and $\mathbf{e} ; 1 \mathrm{~nm}$ in $\mathbf{b}, \mathbf{c}$ and $\mathbf{f} ; 20 \mathrm{~nm}$ in $\mathbf{d}$.

a
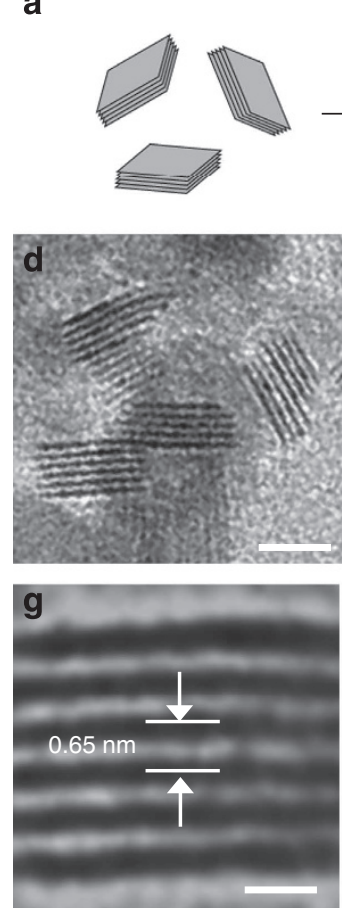

b
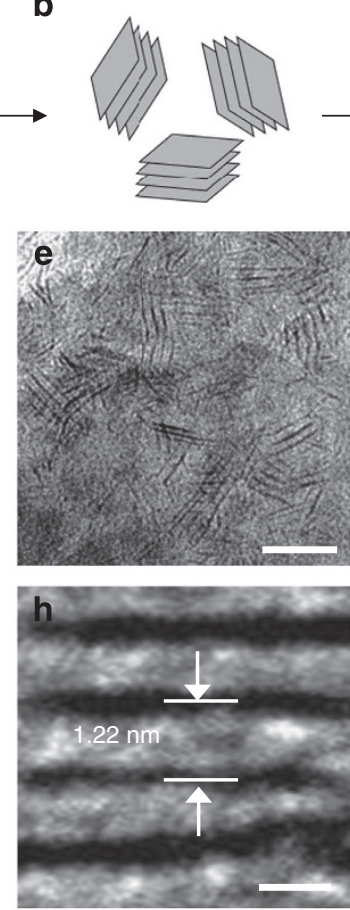

C
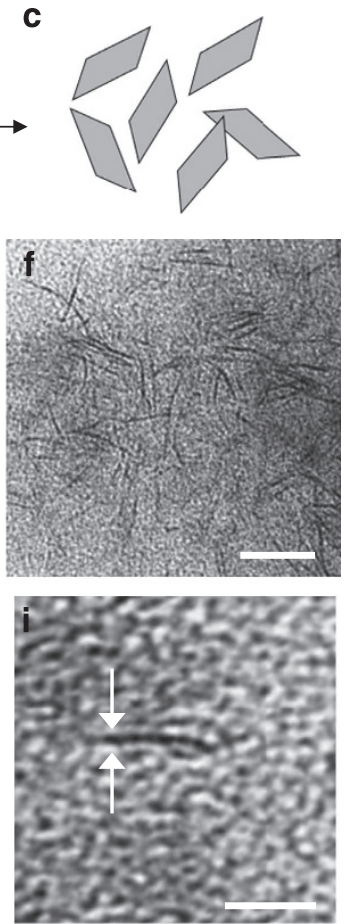

Figure 6 | Exfoliation of multi-layer WSe $\mathbf{e}_{\mathbf{2}}$ nanostructure. (a-c) Schematic illustration of $\mathrm{WSe}_{2}$ exfoliation processes. (d-f) Low-magnification and (g-i) magnified side-view TEM image of starting multi-layer WSe $\mathrm{W}_{2}$ nanostructures $(\mathbf{d}, \mathbf{g}), \mathrm{WSe}_{2}$ intercalated with ethoxide in bilayer arrangement $(\mathbf{e}, \mathbf{h})$ and single-layer $\mathrm{WSe}_{2}$ nanosheets (f,i). Scale bars, $5 \mathrm{~nm}$ in $\mathbf{d}$ and $\mathbf{i} ; 10 \mathrm{~nm}$ in $\mathbf{e}$ and $\mathbf{f} ; 1 \mathrm{~nm}$ in $\mathbf{g}$ and $\mathbf{h}$.
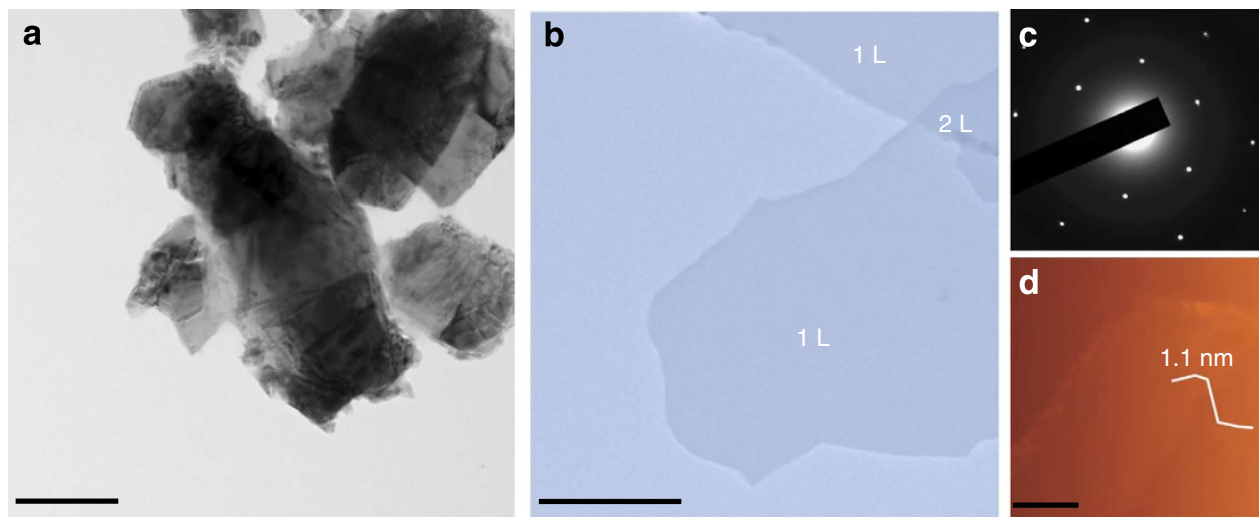

Figure 7 | Micron-size $\mathbf{M o S}_{\mathbf{2}}$ exfoliation by TMI method. (a) TEM image of micron size multi-layer $\mathrm{MoS}_{2}$. (b) TEM image (pseudo-colour) of exfoliated single-layer $\mathrm{MoS}_{2}$ (L, layer). (c) SAED pattern and (d) AFM image of exfoliated $\mathrm{MoS}_{2}$. Scale bars, $1 \mu \mathrm{m}$ in $\mathbf{a} ; 0.5 \mu \mathrm{m}$ in $\mathbf{b} ; 0.1 \mu \mathrm{m}$ in d.

After $9 \mathrm{~h}$, single-layer $\mathrm{ZrS}_{2}$ nanosheets are obtained after centrifugation (Fig. 5d). The thickness measured by TEM is $\sim 6.0 \AA$, which is consistent with the estimated thickness of single-layer $\mathrm{ZrS}_{2}$ (Fig. 5e). A top-view HRTEM image of a singlelayer $\mathrm{ZrS}_{2}$ nanosheet shows lattice fringes with interplanar spacings of 3.1 and $1.8 \AA$, corresponding to the (100) and (110) 
planes of the hexagonal $1 \mathrm{~T}-\mathrm{ZrS}_{2}$, respectively (Fig. 5f and Supplementary Fig. 2c). This TMI method with propyl- and hexylamine intercalates is also effective to group $\mathrm{V} \mathrm{NbS}{ }_{2}$ nanoparticles (Supplementary Fig. 4).

For the exfoliation of multi-layer $\mathrm{WSe}_{2}$ nanostructure, tandem intercalates with high Lewis basicity are chosen. Two differentlength intercalates of sodium ethoxide and sodium hexanolate are added to multi-layer $\mathrm{WSe}_{2}$ nanostructure in DMSO. After stirring for $7 \mathrm{~h}$ at room temperature, single-layer $\mathrm{WSe}_{2}$ nanosheets are isolated by centrifugation. During the course of intercalation, the expansion of the $6.5 \AA$ interlayer spacing of $\mathrm{WSe}_{2}$ (Fig. 6g) to $12.2 \AA$ (Fig. 6h) suggests that ethoxide intercalation proceeds to form a bilayer arrangement between the layers. Figure 6f,i show low-magnification and magnified TEM images of single-layer $\mathrm{WSe}_{2}$ nanosheets after exfoliation, where a side view of standing single-layer $\mathrm{WSe}_{2}$ nanosheets is observed. This exfoliation process can be scaled up to sub-gram scale (Supplementary Fig. 5).

The choice of proper intercalates is critical to have their effective intercalation into TMCs. Intercalates act as Lewis base with highest occupied molecular orbital (HOMO) and TMCs act as Lewis acid with lowest unoccupied molecular orbital (LUMO). Therefore, as the HOMO-LUMO energy difference $\left(\Delta \mathrm{E}_{\text {HOMO-LUMO }}\right)$ is smaller, the more favoured their interaction becomes. Such HOMO-LUMO Lewis acid-base interaction can be generally applied to group IV and VI TMCs. LUMO of group VI TMCs (for example, $\mathrm{MoS}_{2}, \mathrm{MoSe}_{2}, \mathrm{MoTe}_{2}, \mathrm{WS}_{2}, \mathrm{WSe}_{2}$ and $\mathrm{WTe}_{2}$ ) is between -4.2 and $-3.5 \mathrm{eV}$, which is in close energy level with HOMO (approximately $-4.2 \mathrm{eV}$ ) of strong base alkoxides to have favoured interactions between them ${ }^{37,38}$. Similarly, LUMO of group IV TMCs (for example, $\mathrm{TiS}_{2}, \mathrm{ZrS}_{2}$, $\mathrm{ZrSe}_{2}, \mathrm{HfS}_{2}$ and $\mathrm{HfSe}_{2}$ ) is ranging from -6.0 to $-5.5 \mathrm{eV}$, which is close to HOMO (approximately $-6.2 \mathrm{eV}$ ) of relatively weaker base alkylamines ${ }^{37-39}$.

While our TMI method is most effective for the exfoliation of laterally small $(<100 \mathrm{~nm})$ TMC nanoparticles, it can be also extended to larger micron-size TMCs. For the exfoliation of micron-size TMCs (Fig. 7 and Supplementary Fig. 6), sodium ethoxide and hexanolate are added to a solution of $\mathrm{MoS}_{2}$ in DMSO. After stirring $48 \mathrm{~h}$, single-layer $\mathrm{MoS}_{2}$ nanosheets over $1 \mu \mathrm{m}$ are observed (Fig. 7). Similarly, micron-size multilayer TMCs of $\mathrm{WSe}_{2}$ and $\mathrm{TiS}_{2}$ can also be exfoliated (Supplementary Figs 2a and 6). Although specific conditions (for example, time, type and concentration of intercalates, temperature and so on) should be further optimized for efficient exfoliation, this preliminary study indicates the potential of TMI method for large-size TMCs.

\section{Discussion}

In this study, we demonstrate that 'tandem molecular intercalation (TMI)' is an effective new concept for the exfoliation of multi-layer colloidal TMC nanostructures into single-layer TMC nanosheets. TMI is a relatively fast process for producing singlelayer TMCs and is conducted at room temperature, with the clear merit of eliminating the occasional degradation of single-layer TMCs by avoiding harsh exfoliation processes. A variety of Lewis bases can be intercalated into TMC nanostructures, and group IV $\left(\mathrm{TiS}_{2}\right.$ and $\left.\mathrm{ZrS}_{2}\right)$, group $\mathrm{V}\left(\mathrm{NbS}_{2}\right)$ and VI $\left(\mathrm{WSe}_{2}, \mathrm{MoS}_{2}\right)$ TMCs require different types of intercalates. A relatively weaker Lewis base, such as alklyamine, works nicely for group IV and V TMCs, whereas a stronger Lewis base, such as alkoxide, is required for group VI TMCs. In principle, TMI can serve as a general strategy for the exfoliation of wide range of colloidal TMC nanostructures under mild exfoliation conditions, namely, a room-temperature process without sonication or $\mathrm{H}_{2}$ evolution.

\section{Methods}

Alkylamine chain length-dependent intercalation. Propylamine $(0.07 \mathrm{ml}$ $0.91 \mathrm{mmol}$ ), pre-synthesized $\mathrm{TiS}_{2}$ (ref. $40 ; 0.05 \mathrm{mmol}$ ) and DMSO $(5 \mathrm{ml}$ ) are added to a $10 \mathrm{ml}$ vial. The mixture is stirred for $30 \mathrm{~min}$ at room temperature. The mixture is then precipitated by centrifugation at 3,000 r.p.m. for $30 \mathrm{~min}$, and the precipitates are washed three times with chloroform. The intercalation conditions for other alkylamines are identical except for the intercalation time ( $1 \mathrm{~h}$ for butylamine and $5 \mathrm{~h}$ for hexylamine).

Exfoliation of multi-layer TMC nanostructures. Multi-layer colloidal $\mathrm{TiS}_{2}$ nanostructure $(5.0 \mathrm{mg}, 0.04 \mathrm{mmol})^{40}$, propylamine $(3.0 \mathrm{ml}, 36.5 \mathrm{mmol})$, hexylamine $(2.0 \mathrm{ml}, 15.1 \mathrm{mmol})$ and DMSO $(5 \mathrm{ml})$ are mixed to a $10 \mathrm{ml}$ vial and stirred for $10 \mathrm{~h}$ at room temperature. Then, the mixture is centrifuged at 3,000 r.p.m. for $30 \mathrm{~min}$ to precipitate non-exfoliated materials. The supernatant is re-centrifuged at

15,000 r.p.m. for $50 \mathrm{~min}$ to precipitate exfoliated TMCs. Then, chloroform $(10 \mathrm{ml})$ is added to dissolve the precipitate and the resulting purple solution is centrifuged at 17,500 r.p.m. for $10 \mathrm{~min}$. This process repeats several times and then the final product is dried under vacuum at room temperature for $2 \mathrm{~h}$ to afford exfoliated $\mathrm{TiS}_{2}(1.4 \mathrm{mg}, 27 \%)$ as a purple powder.

The exfoliation of multi-layer $\mathrm{ZrS}_{2}$ follows similar procedures. Multi-layer colloidal $\mathrm{ZrS}_{2}$ nanostructure $(5.0 \mathrm{mg}, 0.03 \mathrm{mmol})^{40}$, propylamine $(2.5 \mathrm{ml}$, $30.4 \mathrm{mmol})$, hexylamine $(1.0 \mathrm{ml}, 7.6 \mathrm{mmol})$ and DMSO $(5 \mathrm{ml})$ are mixed to a $10 \mathrm{ml}$ vial and stirred for $9 \mathrm{~h}$ at room temperature. Single-layer $\mathrm{ZrS}_{2}$ (1.6 mg, 32\%) is obtained by following the same purification process of $\mathrm{TiS}_{2}$.

For the exfoliation of WSe $\mathrm{W}_{2}$, multi-layer colloidal WSe $\mathrm{W}_{2}$ nanostructure $(10.0 \mathrm{mg}$ $0.03 \mathrm{mmol})$, sodium ethoxide $(1.50 \mathrm{~g}, 22.0 \mathrm{mmol})$, sodium hexanolate $(0.50 \mathrm{~g}$, $4.03 \mathrm{mmol})$ and DMSO $(15 \mathrm{ml})$ are mixed. After $7 \mathrm{~h}$ stirring, the mixture is centrifuged at 6,000 r.p.m. for $30 \mathrm{~min}$ to precipitate the non-exfoliated materials. Then the supernatant is re-centrifuged at 15,000 r.p.m. for $60 \mathrm{~min}$ to precipitate exfoliated TMCs. The precipitate is washed several times with ethanol via centrifugation at 17,500 r.p.m. for $20 \mathrm{~min}$ to give exfoliated $\mathrm{WSe}_{2}(3.3 \mathrm{mg}, 33 \%)$.

Instruments. TEM and HRTEM analyses were performed using JEM 2100 at $200 \mathrm{kV}$ and JEOL-ARM1300S at $1,250 \mathrm{kV}$, respectively. Atomic force microscopy (AFM) images were obtained in non-contact mode using a scanning probe microscope (Veeco). X-ray diffraction (XRD) studies were conducted using Rigaku-G2005304 equipped with a $\mathrm{Cu}_{\mathrm{k} \alpha}$ radiation source $(30 \mathrm{kV}, 15 \mathrm{~mA})$. ${ }^{1} \mathrm{H}$-NMR spectra were obtained using a $400 \mathrm{MHz}$ FT-NMR Spectrometer (Bruker Biospin).

Density-functional theory calculations. The DFT calculations were performed using the Amsterdam Density-Functional (ADF2013-BAND) package ${ }^{41,42}$. We used the local VWN exchange-correlation potential with nonlocal PerdewBurke-Ernzerhof exchange-correlation correction and empirical D3 treatment of London dispersion interactions (PBE-D3-BJ $)^{43}$.

\section{References}

1. Yoffe, A. D. Layer compounds. Annu. Rev. Mater. Sci. 3, 147-170 (1973)

2. Frienda, R. H. \& Yoffe, A. D. Electronic properties of intercalation complexes of the transition metal dichalcogenides. Adv. Phys. 36, 1-94 (1987).

3. Jaramillo, T. et al. Identification of active edge sites for electrochemical $\mathrm{H}_{2}$ evolution from $\mathrm{MoS}_{2}$ nanocatalysts. Science 317, 100-102 (2007).

4. Wang, Q. H., Kalantar-Zadeh, K., Kis, A., Coleman, J. N. \& Strano, M. S. Electronics and optoelectronics of two-dimensional transition metal dichalcogenides. Nat. Nanotechnol. 7, 699-712 (2012).

5. Lukowski, M. A. et al. Enhanced hydrogen evolution catalysis from chemically exfoliated metallic $\mathrm{MoS}_{2}$ nanosheets. J. Am. Chem. Soc. 135, 10274-10277 (2013).

6. Splendiani, A. et al. Emerging photoluminescence in monolayer $\mathrm{MoS}_{2}$. Nano Lett. 10, 1271-1275 (2010).

7. Mak, K. F., Lee, C., Hone, J., Shan, J. \& Heinz, T. F. Atomically thin $\mathrm{MoS}_{2}$ : a new direct-gap semiconductor. Phys. Rev. Lett. 105, 136805-1-136805-4 (2010).

8. Kuc, A., Zibouche, N. \& Heine, T. Influence of quantum confinement on the electronic structure of the transition metal sulfide $\mathrm{TS}_{2}$. Phys. Rev. B 83, 245213-1-245213-4 (2011).

9. Lin, L. et al. Fabrication of luminescent monolayered tungsten dichalcogenides quantum dots with giant spin-valley coupling. ACS Nano 7, 8214-8223 (2013).

10. Novoselov, K. S. et al. Two-dimensional atomic crystals. Proc. Nat Acad. Sci. USA 102, 10451-10453 (2005).

11. Radisavljevic, B., Whitwick, M. B. \& Kis, A. Integrated circuits and logic operations based on single-layer $\mathrm{MoS}_{2}$. ACS Nano 5, 9934-9938 (2011).

12. Lin, L. et al. Fabrication and luminescence of monolayered boron nitride quantum dots. Small 10, 60-65 (2014).

13. Lin, L. \& Zhang, S. Creating high yield water soluble luminescent graphene quantum dots via exfoliating and disintegrating carbon nanotubes and graphite flakes. Chem. Commun. 48, 10177-10179 (2012). 
14. Dines, M. B. Lithium interacalation via n-butyllithium of the layered transition metal dichalcogenides. Mater. Res. Bull. 10, 287-292 (1975).

15. Joensen, P., Frindt, R. F. \& Morrison, S. R. Single-layer $\mathrm{MoS}_{2}$. Mater. Res. Bull. 21, 457-461 (1986)

16. Eda, G. et al. Photoluminescence from chemically exfoliated $\mathrm{MoS}_{2}$. Nano Lett. 11, 5111-5116 (2011).

17. Zheng, J. et al. High yield exfoliation of two-dimensional chalcogenides using sodium naphthalenide. Nat. Commun. 5, 2995 (2014).

18. Zeng, Z. et al. An effective method for the fabrication of few-layer-thick inorganic nanosheets. Angew. Chem. Int. Ed. 51, 9052-9056 (2012).

19. Yuwen, L. et al. General synthesis of noble metal (Au, Ag, Pd, Pt) nanocrystal modified $\mathrm{MoS}_{2}$ nanosheets and the enhanced catalytic activity of $\mathrm{Pd}-\mathrm{MoS}_{2}$ for methanol oxidation. Nanoscale 6, 5762-5769 (2014).

20. Bissessur, R., Kanatzidis, M. G., Schindlerb, J. L. \& Kannewurf, C. R. Encapsulation of polymers into $\mathrm{MoS}_{2}$ and metal to insulator transition in metastable $\mathrm{MoS}_{2}$. J. Chem. Soc. Chem. Commun. 1582-1585 (1993).

21. Gordon, R. A., Yang, D., Crozier, E. D., Jiang, D. T. \& Frind, R. F. Structures of exfoliated single layers of $\mathrm{WS}_{2}, \mathrm{MoS}_{2}$, and $\mathrm{MoSe}_{2}$ in aqueous suspension. Phys. Rev. B 65, 125407-1-125407-9 (2002).

22. Coleman, J. N. et al. Two-dimensional nanosheets produced by liquid exfoliation of layered materials. Science 331, 568-571 (2011).

23. Zhou, K.-G., Mao, N.-N., Wang, H.-X., Peng., Y. \& Zhang, H.-L. A mixedsolvent strategy for efficient exfoliation of inorganic graphene analogues. Angew. Chem. Int. Ed. 50, 10839-10842 (2011).

24. Smith, R. J. et al. Large-scale exfoliation of inorganic layered compounds in aqueous surfactant solutions. Adv. Mater. 23, 3944-3948 (2011).

25. Mashtalir, O. et al. Intercalation and delamination of layered carbides and carbonitrides. Nat. Commun. 4, 1716 (2013).

26. O’Neill, A., Umar, K. \& Coleman, J. N. Preparation of high concentration dispersions of exfoliated $\mathrm{MoS}_{2}$ with increased flake size. Chem. Mater. 24, 2414-2421 (2012).

27. Gamble, F. R. et al. Intercalation complexes of Lewis bases and layered sulfides: a large of new superconductors. Science 174, 493-497 (1971).

28. Levy, F. Intercalated layered materials (Springer Link, 1979).

29. Chianelli, R. R., Scanlon, J. C. \& Thompson, A. H. Structure refinement of stoichiometric $\mathrm{TiS}_{2}$. Mater. Res. Bull. 10, 1379-1382 (1975).

30. Lagaly, G. Intercalation of alkylamines with different types of layered compounds. Solid State Ion 22, 43-51 (1986).

31. Whittingham, M. S. \& Chianelli, R. R. Layered compounds and intercalation chemistry: an example of chemistry and diffusion in solids. J. Chem. Educ. 57, 569-574 (1980).

32. Ulman, A., Eilers, J. E. \& Tillman, N. Packing and molecular orientation of alkanethiol monolayers on gold surfaces. Langmuir 5, 1147-1152 (1989).

33. Sieval, A. B., Hout, B. V. D., Zuilhof, H. \& Sudhölter, E. J. R. Molecular modeling of covalently attached alkyl monolayers on the hydrogen-terminated Si(111) surface. Langmuir. 17, 2172-2181 (2007).

34. Zhang, R.-Z., Wan, C.-L., Wang, Y.-F. \& Koumoto, K. Titanium sulphene: twodimensional confinement of electrons and phonons giving rise to improved thermoelectric performance. Phys. Chem. Chem. Phys. 14, 15641-15644 (2012).
35. Lin., C. et al. Hydrogen-incorporated $\mathrm{TiS}_{2}$ ultrathin nanosheets with ultrahigh conductivity for stamp-transferrable electrodes. J. Am. Chem. Soc. 135, 5144-5151 (2013).

36. Li, Y., Kang, J. \& Li, J. Indirect-to-direct band gap transition of the $\mathrm{ZrS}_{2}$ monolayer by strain: first-principles calculations. RSC Adv. 4, 7396-7401 (2014).

37. Gong, C. et al. Band alignment of two-dimensional transition metal dichalcogenides: application in tunnel field effect transistors. Appl. Phys. Lett. 103, 053513-1-053513-4 (2013).

38. NIST Standard Reference Database Number 101. http://cccbdb.nist.gov/ (accessed 25 August 2014).

39. Miro, P., Han, J. H., Cheon, J. \& Heine, T. Hexagonal transition-metal chalcogenide nanoflakes with pronounced lateral quantum confinement. Angew. Chem. Int. Ed. 53, 12624-12628 (2014).

40. Jeong, S., Yoo, D., Jang, J.-t., Kim, M. \& Cheon, J. Well-defined colloidal 2-D layered transition-metal chalcogenide nanocrystals via generalized synthetic protocols. J. Am. Chem. Soc. 134, 18233-18236 (2012).

41. Velde, G. T. \& Baerends, E. J. Precise density-functional method for periodic structures. Phys. Rev. B 44, 7888-7903 (1991).

42. Wiesenekker, G. \& Baerends, E. J. Quadratic integration over the threedimensional Brillouin zone. J. Phys. Condens. Mater. 3, 6721-6742 (1991).

43. Perdew, J. P., Burke, K. \& Ernzerhof, M. Generalized gradient approximation made simple. Phys. Rev. Lett. 77, 3865-3868 (1996).

\section{Acknowledgements}

This work was financially supported by the National Creative Research Initiatives Program (2010-0018286).

\section{Author contributions}

S.J., D.Y. and J.C. designed the experiments and analysed the data. S.J. and M.A. performed the synthesis and characterization. S.J., D.Y. and J.C. wrote the manuscript. P.M. and T.H. performed the density-functional theory calculations. All the authors discussed the results and commented on the manuscript.

\section{Additional information}

Supplementary Information accompanies this paper at http://www.nature.com/ naturecommunications

Competing financial interests: The authors declare no competing financial interests.

Reprints and permission information is available online at http://npg.nature.com/ reprintsandpermissions/

How to cite this article: Jeong, S. et al. Tandem intercalation strategy for single-layer nanosheets as an effective alternative to conventional exfoliation processes. Nat. Commun. 6:5763 doi: 10.1038/ncomms6763 (2015). 